\title{
Selected Abstracts From the Proceedings of the 2015 Society of Teachers of Family Medicine (STFM) Conference on Medical Student Education
}

\author{
Mustafa Alavi, MD | Katherine Margo, MD
}

PRIMER. 2019;3:4.

Published: 2/5/2019 | DOI: 10.22454/PRiMER.2019.564543

\section{Abstract}

The Society of Teachers of Family Medicine (STFM; http://www.stfm.org) is a community of professionals devoted to teaching family medicine through undergraduate, graduate, and continuing medical education. This multidisciplinary group of physicians, educators, behavioral scientists, and researchers works to further STFM's mission of improving the health of all people through education, research, patient care, and advocacy. The STFM held its 41st Conference on Medical Student Education in Atlanta, Georgia from February 5-8, 2015. The conference this year was met with record attendance and student scholarship winners. The STFM Education Committee selected 10 abstracts, of which five are presented below.

Editor's Note: The following five of the 10 selected abstracts have been published in the intervening time, and are not included in this publication:

1. Biagioli FE, Elliot DL, Palmer RT, et al. The electronic health record objective structured clinical examination: assessing student competency in patient interactions while using the electronic health record. Acad Med. 2017;92(1):87-91. https://doi.org/10.1097/ACM.0000000000001276

2. Mader EM, Rodríguez JE, Campbell KM, et al. Status of underrepresented minority and female faculty at medical schools located within historically black colleges and in Puerto Rico. Med Educ Online. 2016;21(1):29535. https://doi.org/10.3402/meo.v21.29535

3. Phillips JP, Wendling AL, Fahey CA, Mavis BE. The effect of a community-based medical school on the state and local physician workforce. Acad Med. 2017.

4. White J, Anthony D, WinklerPrins V, Roskos S. WinklerPrins V, Roskos S. Electronic medical records, medical students, and ambulatory family physicians: a multi-institution study. Acad Med. 2017;92(10):1485-1490. https://doi.org/10.1097/ACM.0000000000001673

5. Morley CP, Rosas SR, Mishori R, et al. Essential public health competencies for medical students: establishing a consensus in family medicine. Teach Learn Med. 2017;29(3):255-267. https://doi.org /10.1080/10401334.2016.1268964 


\section{Development and Validation of a Biopsychosocial Integrated Standardized Patient Examination (ISPE) for the Family Medicine Clerkship}

\section{Christopher P. Morley (morleycp@upstate.edu)}

Department of Family Medicine, Department of Public Health and Preventive Medicine, and Department of Psychiatry and Behavioral Sciences, SUNY Upstate Medical University, Syracuse, NY

Background: Communication, especially with socially complex patients, is a skill that tends to receive less emphasis in medical school in the United States. ${ }^{1,2}$ In order to assess communication skills with socially and medically complex patients, the Integrated Standardized Patient Examination (ISPE) was developed, implemented, and evaluated in a family medicine (FM) clerkship.,4 The current study examined the score distribution and convergent validity in a larger sample than used for the initial evaluation.

Methods: In order to assess the distribution of ISPE scores, and compare the distribution with preceptor assessments, the scores from the ISPE and from two preceptor rating instruments were plotted on histograms. To compare the ability of the ISPE to detect poorly performing students relative to other assessments of similar skill domains, scores on the FM clerkship final examination, participation scores, and preceptor-assigned scores were compared between students who passed and failed the ISPE (1/0, failure on ISPE defined as $<65 \%)$ via $t$ test.

Results: A total of 217 individual ISPE responses were available for the analyses. ISPE scores had a mean score of slightly over 77 on a 100-point scale, and a nearly normal distribution, as compared to scores assigned by clinical preceptors, which tend to grade the majority of students well. The ISPE also displays convergent validity, as students who fail the ISPE do significantly poorer on the FM final exam $(P=.003)$, participation score $(P<.001)$, and the preceptor-assigned score $(P=.003)$.

Conclusions: The ISPE performed well, compared with other components of the grade assigned to FM clerkship students. Adaptation in contexts other than FM clerkship, and at other institutions, should be explored. Additionally, differences in grade distribution between structured, observed, and standardized student-patient exercises and preceptor assessments warrant further exploration.

\section{References}

1. Yedidia MJ, Gillespie CC, Kachur E, et al. Effect of communications training on medical student performance. JAMA. 2003;290(9):1157-1165. https://doi.org/10.1001/jama.290.9.1157

2. Howard T, Jacobson KL, Kripalani S. Doctor talk: physicians' use of clear verbal communication. J Health Commun. 2013;18(8):991-1001. https://doi.org/10.1080/10810730.2012.757398

3. Morley CP, Flad JR, Arthur M, et al. Pilot evaluation of a biopsychosocial integrated standardized patient examination in a family medicine clerkship. Int J Psychiatry Med.

2011;41(4):309-328. http://www.ncbi.nlm.nih.gov/pubmed/22238837. Accessed February 26, 2013. https://doi.org/10.2190/PM.41.4.b

4. Manyon AT, Morley CP, Bailey RE, Arthur ME, Recker-Hughes C, Flad JR. First Draft - Integrated Standardized Patient Checklist -SUNY Upstate Family Medicine Clerkship. STFM Resource Library. http://fmdrl.org /index.cfm?event=c. beginBrowseD\&clearSelections=1\&criteria=ISPE\#2927. Published 2010. Accessed February 5, 2014. 


\title{
Patients as Teachers Student Clinic: Third-Year Medical Students' Experiences and Outcomes With a Novel Approach to the Family Medicine Outpatient Clerkship
}

\author{
Sara Oberhelman \\ Assistant Professor of Family Medicine, Department of Family Medicine, Mayo Clinic, Rochester MN \\ Kurt Angstman \\ Associate Professor of Family Medicine, Department of Family Medicine, Mayo Clinic, Rochester MN
}

Background: Outpatient family medicine experiences are an essential aspect of medical student education. Classic models for third-year medical student outpatient clerkships often involve partnering a student with a physician preceptor. In this model, students are often limited to shadowing opportunities and brief opportunities to interview/examine a patient independently due to the time constraints of a full clinical schedule.

Methods: To optimize the outpatient medical student clerkship experience, we developed the Patients as Teachers (PAT) Student Clinic. Each day three students and one faculty preceptor are scheduled in the PAT clinic. Nine patient appointments are available, allowing each student to take responsibility for three patients' care. The student obtains a history, performs an exam, develops a differential/plan, writes a note, places orders, writes prescriptions, and if appropriate, follows up on lab/study results. Each student spends approximately half of their rotation days in the PAT clinic and the remaining half with a one-on-one preceptor, participating in that preceptor's normal clinic schedule. We collected subjective data from students, preceptors, and patients. Students completed patient logs with level of involvement in care.

Results: Forty-one students completed the surveys with $92.7 \%$ reporting a positive educational experience and $85.4 \%$ recommending this model to other rotations. Students identified a statically significant $(P<0.01)$ increased opportunity to independently interview ( $46 \%$ vs $35 \%$ ) and examine ( $46 \%$ vs $37 \%$ ) patients in the PAT clinic as opposed to with their individual preceptor. Student comments exemplified the PAT clinic as an opportunity to take ownership of patients, develop assessments/plans and develop skills necessary for the Step 2 Clinical Skills examination.

Conclusions: Students were often eager to take on more autonomy with patient care. This creative scheduling was able to offer the medical students greater clinical and care management responsibilities while providing excellent exposure to family medicine and primary care teaching.

\section{Win-Win: Student Health Coaching for Early Workplace Learning}

\author{
Paul Marcus, Christina Cicoletti, Jennifer Crawford, Margaret McNamara, and Margo Vener \\ (Margo.Vener@ucsf.edu) \\ The University of California at San Francisco, San Francisco, CA
}

Background: First-year medical students with health coaching skills can counsel patients while freeing up preceptors for other clinical tasks. Patients may experience improved communication and students may experience more meaningful roles in the interdisciplinary team.

Methods: In 2013-2014, 154 first-year medical students participated in health coach training in a 4-hour workshop followed by a 2-hour booster session. The training focused on motivational interviewing, medication reconciliation, and effective patient education strategies. A subset of the students $(n=12)$ worked as health coaches in an urban resident continuity clinic. Students paired with residents and observed the initial examination. Residents presented to attendings, while students discussed health coaching topics with patients (dieting, diabetes education, smoking 
cessation, etc) chosen by residents. Afterwards, students updated the residents on action plans made. IRB approval was obtained.

Results: All students trained as health coaches were surveyed ( $1=$ strongly disagree, $5=$ strongly agree) and felt the workshop was valuable to their education $3.90(0.87)$ and that health coaching was a valuable clinical skill 4.43 (0.68). The 12 students in the resident clinic were surveyed ( $1=$ not confident, $5=$ highly confident) and initially stated their confidence performing health coaching tasks was $3.26(0.18)$. This improved to $4.12(0.16)$ after the sessions finished. A subset of these students $(n=8)$ participated in a focus group identifying three themes: ( 1 ) health coaching increased students' confidence and initiative, (2) residents were excellent teachers, and (3) students valued "making a difference." In surveys ( $1=$ strongly disagree, $5=$ strongly agree) preceptors felt overall visit efficiency improved with health coaches, $4.00(0.11)$. Challenges identified included coordinating student and resident schedules, finding adequate space, and ensuring team members knew student roles.

Conclusions: In spite of logistical challenges, students and preceptors were very satisfied with health coaches' training. We recommend other schools consider this innovation because it synergizes student learning, preceptor time, and patient care.

\section{Interactive Theatre: A Teaching Method for Health Care Professionals, Students and Patients}

\section{Pamela L. Ostby, James D. Campbell (Campbellj@health.missouri.edu), Suzanne Burgoyne, Heather Carver University of Missouri-Columbia, Columbia, MO}

Background: A forum for the exchange of views is an effective way of gaining insight into dilemmas faced by patients and health professionals. Due to the rapid changes in health care and challenges on scientific, technological, and interpersonal levels, the traditional approach to classroom content presentation may not be the most effective method of facilitating transformative learning. The objective of this study was to pilot an interactive theatre (ITT) program to educate and promote transformative discussion with health professionals, students, and the community about the patient-doctor relationship in breast cancer.

Methods: Based on ethnographic interviews, three ITT scenarios were developed illustrating different aspects of the patient-doctor relationship, centered on delivering a cancer diagnosis and treatment. ITT engages the audience through talkback discussion with the characters. Scenarios were written as unresolved stories, creating a collective problem-solving experience. Audience members can also replace a character on stage to try out their own strategy to improve the relationship. Survey development for this study has institutional review board approval.

Results: Eight performances were given to a total of 391 participants, including 116 first-year medical students, 215 nursing students, 35 social work students and 25 breast cancer survivors and health professionals. Results of postperformance 5-point surveys showed positive audience responses with an average rating of $4.5(\mathrm{n}=391)$. Students perceived the material to be suitable for presentation in this format (average rating of 4.5), with a high degree of relevance to their future careers (average rating of $4.1 ; n=366$ ). In open-ended responses, students specifically appreciated the opportunity to learn in a more engaging manner compared to didactic lecture.

Conclusions: ITT is an effective form of active transformational learning. It provokes dialogue while promoting change through interactions between the audience members and the ITT characters.

\section{Professional Formation in a Longitudinal Small Group Format}

\section{Sarah Swofford (swoffords@health.missouri.edu), Kevin Kane, Elizabeth Garrett}

University of Missouri School of Medicine, Columbia, MO

Background: Medical educators not only ensure medical students' knowledge and clinical competency, but also 
prepare them to lead lives of service to their patients and communities. This "professional formation," the moral and professional development of students, is key. The goal of professional formation is to anchor students to foundational principles while helping them navigate the inevitable moral conflicts in medical practice.

Methods: COMPASS (Contemplating Medicine, Patients, Self, and Society), a 4-year longitudinal course, uses innovative blended small group learning experiences composed of medical students from all 4 years of medical school and faculty guides to explore professional formation through a variety of learning strategies including reflective writing, storytelling, readings, discussions, and case problem solving. COMPASS also facilitates the formation of longitudinal relationships among medical students across classes and course faculty. Over 70 faculty from 19 departments volunteer to facilitate these groups and share their vast professional experience and stories.

Results: Session topics during the first 2 years included stress and coping strategies, personal/professional choices and boundaries, the cultural self, transitions, physician values, implicit bias, and patient advocacy. Fifty to $80 \%$ of students agreed or strongly agreed the session content was important to their professional development. Students enjoy interacting with students from other classes and appreciate space for discussing sensitive topics. They prefer less structured content and more time for spontaneous discussion.

Conclusions: It is too early to judge the success of COMPASS since no student has participated in all 4 years of the course. However, preliminary data show a majority of students rate the experience as important to their professional development. Having key stakeholders involved in the initial and subsequent planning and implementation helped to overcome the challenges of integrating this course into an already existing curriculum. Faculty and dean's office support and resources are keys to success.

\section{Corresponding Author}

Mustafa Alavi, MD

Oregon Health and Science University, Department of Family Medicine, 3181 SW Sam Jackson Park Rd, Mail Code: FM, Portland, OR 97239

mustafa.alavi18@gmail.com

\section{Author Affiliations}

Mustafa Alavi, MD - Department of Family Medicine, Oregon Health and Science University, Portland, OR Katherine Margo, MD - Department of Family Medicine and Community Health, Perelman School of Medicine at the University of Pennsylvania, Philadelphia, PA

Copyright $@ 2019$ by the Society of Teachers of Family Medicine 